\title{
Accelerated Idioventricular Rhythm and Hypokalemia, Atypical Presentation in the Midst of a Pandemic
}

\author{
Carlos Rodríguez-Artuza1,2,*, Mayela Labarca Torres², José Meza²
}

\author{
ORCID IDs \\ Rodríguez-Artuza C (D) https://orcid.org/0000-0001-7925-6145 \\ Labarca Torres M (D) https://orcid.org/0000-0002-2789-3537
}

\begin{abstract}
A 34-year-old female patient was referred to a private center from a rural population due to exertional dyspnea and palpitations for 15 days being previously asymptomatic, a surface electrocardiogram diagnoses sinus rhythm alternating with accelerated idioventricular rhythm with episodes of short-coupling intervals. When conducting a diagnostic investigation, hypokalemia and decreased serum magnesium were evidenced and, after correction of the water-electrolyte imbalance, the electrocardiographic disorders disappeared.
\end{abstract}

KEYWORDS: Slow ventricular tachycardia; Short-coupling interval; Water-electrolyte imbalance.

\section{INTRODUCTION}

Accelerated idioventricular rhythm (AIVR) is a rhythm of ventricular origin generating from the His bundle, the Purkinje system or the ventricular myocyte that consists of three or more consecutive, monomorphic, rarely polymorphic, slow and gradual onset beats, with a rate that can vary between 50 and 120 beats per minute (bpm). Accelerated idioventricular rhythm occurs when the sinus rate falls below the rate of the ectopic focus or when the rate of the focus accelerates above the sinus rate ${ }^{1}$. On the other hand, hypokalemia results in a decrease in the ventricular repolarization reserve and promote the development of ventricular arrhythmias mediated by early late postpotentials that generate polymorphic ventricular tachycardia, torsade de pointes and

1. Universidad del Zulia - Facultad de Medicina - Instituto de Investigaciones de Enfermedades Cardiovasculares - Maracaibo/Zulia Venezuela.

2. Centro Clínico La Sagrada Familia - Medicine - Cardiology - Maracaibo/Zulia - Venezuela.

*Corresponding author: cardiocarral@gmail.com

Received: Sep 21, 2020 | Accepted: Feb 22, 2021 
ventricular fibrillation ${ }^{2}$. Up until now, we haven't found reports of accelerated idioventricular rhythm associated with hypokalemia in humans.

\section{CASE PRESENTATION}

A 34-year-old female patient, who is referred from a rural population $3 \mathrm{~h}$ from the city of Maracaibo, complaining of palpitations and dyspnea on exertion for approximately 15 days, denies syncope, chest pain, diarrhea, vomiting, polyuria, polyphagia, muscle pain, tremors, high blood pressure, diabetes, chronic obstructive pulmonary disease (COPD) or myocardial infarction. She referred that an aunt suffered sudden death at the age of 25 as a result of a fright, according to her mother. On physical examination, neurological status is preserved. Neck: nonpalpable thyroid, without masses or jugular engorgement. Blood pressure of 120/80 $\mathrm{mmHg}$, heart rate $117 \mathrm{bpm}$. Normal heartbeat sounds, no murmurs, normal apex beat position. Eupneic, 99\% oxygen saturation, vesicular murmur present in both lung fields without rales. Soft, nonpainful, depressible abdomen without palpable masses. Lower limbs without edema and symmetrical pulses present. The patient does not bring any paraclinical study. We immediately performed 12-lead surface electrocardiogram (ECG) where we evidenced accelerated idioventricular rhythm with short-coupling beats, electrocardiographically originating in the left ventricle in the anterolateral papillary muscle area alternating with episodes of sinus rhythm (Figs. 1 and 2).

Hospital admission was indicated; the patient refused due to the COVID-19 pandemic and fear of getting infected, owing to the lack of logistics in the city taking into account the quarantine in which we are immersed. We immediately decided to proceed requesting laboratory tests and, while we awaited the results, we began our inquest.

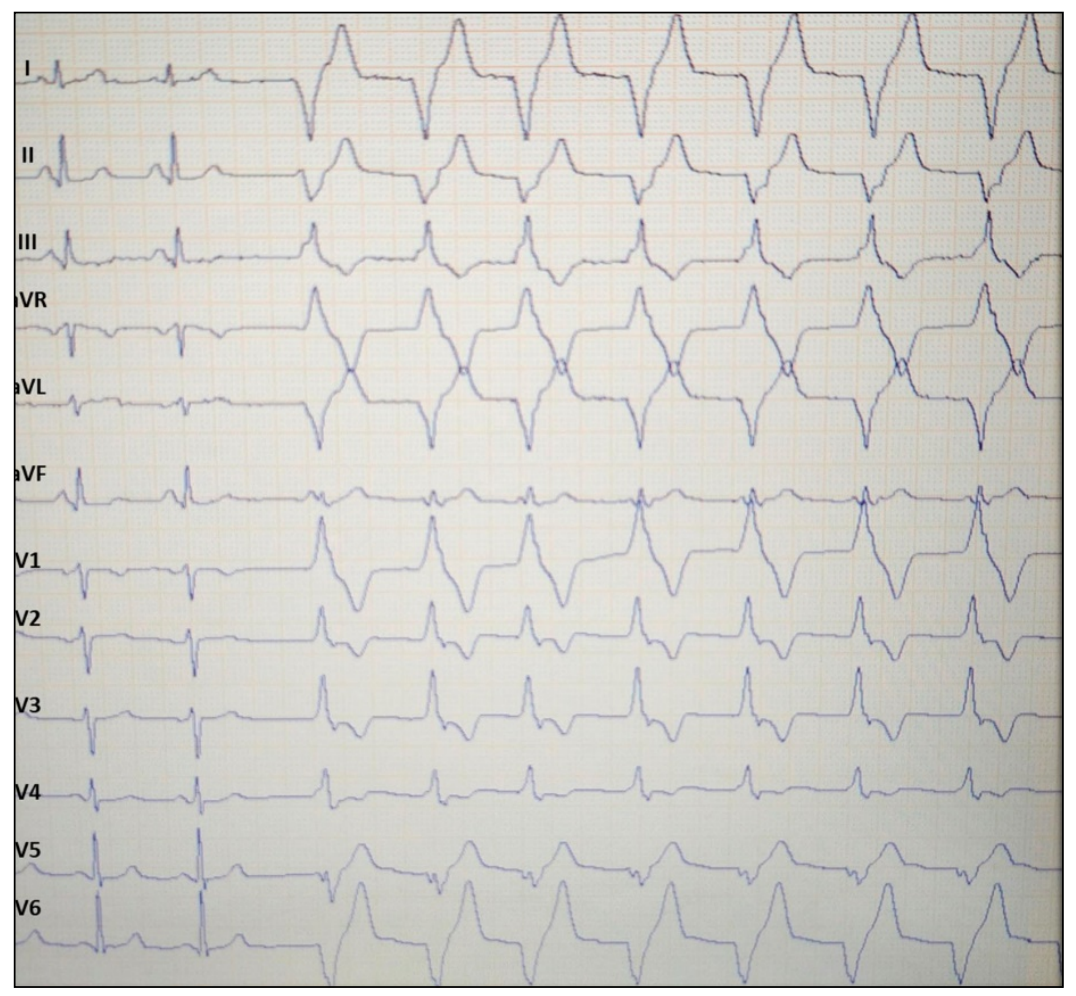

Figure 1. Sinus rhythm alternating with AIVR. 


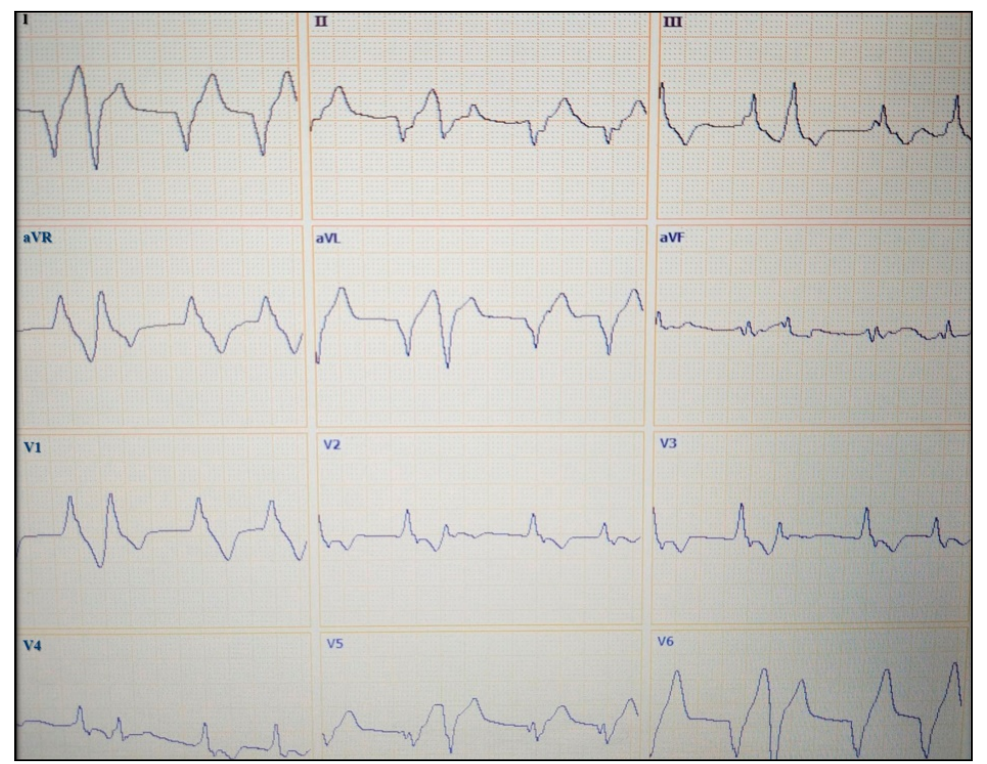

Figure 2. Accelerated idioventricular rhythm with short-coupling beats.

\section{DISCUSSION}

The idioventricular rhythm is described in patients with or without structural heart disease $e^{3,4}$, which is why we performed an echocardiogram; there we observed anatomical concordance and cardiac cavities within a normal range with a normal ejection fraction. Once structural cardiac condition had been ruled out and without the laboratory tests in our hands, even with the evidence of accelerated idioventricular rhythm with episodes of the focus presenting short coupling, we decided to perform a stress test, since the patient in the real-world wanders through the streets with this rhythm and the ergometric test is an essay that helps to assess sinus node disease and cardiac ischemia that are recognized as conditions that cause AIVR ${ }^{3-5}$. We performed an ergometric test according to the original Bruce protocol and, at the end of the first stage, we evidenced the disappearance of accelerated idioventricular rhythm, while sinus rhythm assumed command with excellent chronotropic response and a negative result for cardiac ischemia (Fig. 3), thus ruling out ischemic cause and sinus node disease as triggers for AIVR.

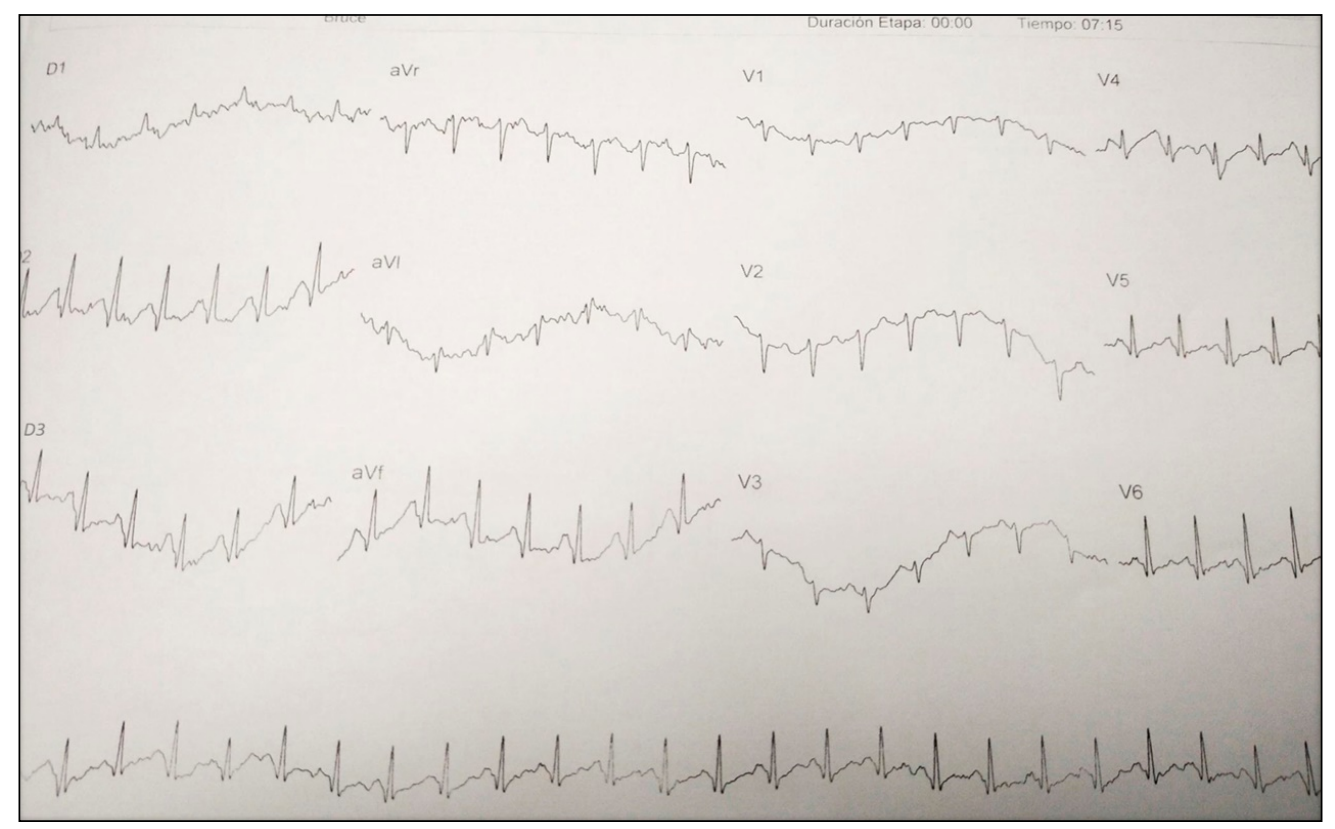

Figure 3. Negative stress test for ischemia and disappearance of AIVR. 
During the interview, the patient denied taking medication, which has also been implicated as a cause of accelerated idioventricular rhythm ${ }^{1,6}$.

After performing echocardiogram and ergometric test, we noticed an electrolyte disturbance on the laboratory report, a potassium level of 3 milliequivalents and magnesium level of $1.2 \mathrm{mg}$. The rest of the studies, including thyroid tests, were within the normal range. Water-electrolyte imbalance is described as causing accelerated idioventricular rhythm in experimental animals; however, there are no cases reported in humans ${ }^{7}$, this patient was treated with spironolactone, $1500 \mathrm{cc}$ intravenous normal saline plus $2 \mathrm{~g}$ of magnesium and 120 milliequivalents of potassium in $24 \mathrm{~h}$. After the correction of potassium and magnesium imbalances, the patient became asymptomatic and the AIVR disappeared (Fig. 4).

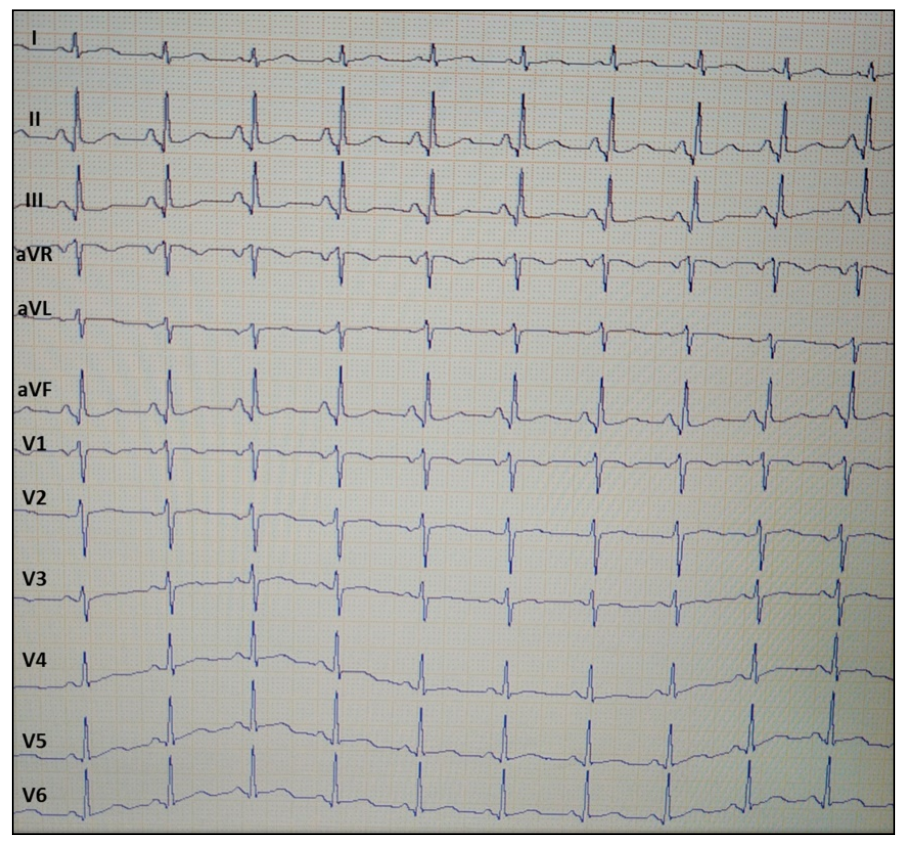

Figure 4. Sinus rhythm restoration after electrolyte imbalance correction.

The patient has a history of sudden first-degree family death; idioventricular rhythm has been reported as the first manifestation to unmask some channelopathies, such as Brugada syndrome ${ }^{8}$. Even though hypokalemia can unmask long QT syndrome, we did not observe long QT in this patient, nor did we observe short QT. In the surface ECG, we evidenced criteria of early ventricular repolarization in the inferior wall, which could explain the episodes of AIVR with short-coupled ventricular beats and the potential risk of triggering ventricular fibrillation ${ }^{9}$. During the correction of the electrolyte imbalance, we associated cilostazol for prevention of ventricular fibrillation ${ }^{10}$. We believe that the patient did not develop a malignant ventricular tachycardia, such as polymorphic ventricular tachycardia, torsade de pointes or ventricular fibrillation, because potassium levels were not reduced below 3 milliequivalents and the accelerated idioventricular rhythm is a benign rhythm that protected this patient.

Currently, the patient persists asymptomatic and is being evaluated on an outpatient basis by the departments of internal medicine, nephrology and cardiology to investigate the etiology of the electrolyte disturbance.

\section{CONCLUSION}

Accelerated idioventricular rhythm is a generally benign course rhythm that must be taken into account in all patients who evolve with fluid and electrolyte imbalances. 


\section{ACKNOWLEDGMENTS}

Not applicable.

\section{FUNDING}

Not applicable.

\section{AUTHORS' CONTRIBUTION}

Conceptualization: Rodríguez-Artuza C. and Meza J.; Investigation: Rodríguez-Artuza C. and Meza J.; Writing - Original Draft: Rodríguez-Artuza C. and Meza J.; Writing - Review and Editing: Rodríguez-Artuza C. and Labarca Torres M.; Supervision: Rodríguez-Artuza C.

\section{REFERENCES}

1. Rodríguez-Artuza CR, Rivera J, Gutiérrez L, González K, Chacón J, Madueño F. Ritmo idioventricular acelerado en escolar de 10 años. Avances Cardiol. 2011;31(3):270-1.

2. Pezhouman A, Singh N, Song Z, Nivala M, Eskandari A, Cao H, et al. Molecular basis of hypokalemia-induced ventricular fibrillation. Circulation. 2015;132(16):1528-37. https://doi.org/10.1161/circulationaha.115.016217

3. Riera ARP, Barros RB, Sousa FD, Baranchuk A. Accelerated idioventricular rhythm: history and chronology of the main discoveries. Indian Pacing Electrophysiol J. 2010;10(1):40-8.

4. Honarbakhsh S, Suman-Horduna I, Mantziari L, Ernst S. Grouped beating in Eisenmenger: Successful localization and ablation of an accelerated idioventricular rhythm from within the Purkinje system. Indian Pacing Electrophysiol J. 2013,13(3):126-30. https://doi. org/10.1016/S0972-6292(16)30631-3

5. Assefa M, Yasar SJ, Abdullah O, Fong HK, Balla S. Accelerated idioventricular rhythm at the termination of an episode of vasospastic angina. Cureus. 2019;11(1):e3895. https://doi.org/10.7759/cureus.3895

6. Jakkoju A, Jakkoju R, Subramaniam PN, Glancy L. Accelerated idioventricular rhythm. Proc (Bayl Univ Med Cent). 2018;31(4):506-7. https://doi.org/10.1080/08998280.2018.1493323

7. Moroz VM, Lipnitskii TN. Study of pathogenesis of ventricular arrhythmia in experimental rats by separation of sinus and ventricular substitutional rhythms. Bull Exp Biol Med. 2006;141(4):400-3. https://doi.org/10.1007/s10517-006-0182-4

8. Elizari MV, Conde D, Baranchuk A, Chiale PA. Accelerated idioventricular rhythm unmasking the Brugada electrocardiographic pattern. Ann Noninvasive Electrocardiol. 2015;20(1):91-3. https://doi.org/10.1111/anec.12177

9. Antzelevitch C, Barajas-Martinez H. A gain-of-function IK-ATP mutation and its role in sudden cardiac death associated with J-wave syndromes. Heart Rhythm. 2010;7(10):1472-4. https://doi.org/10.1016/j.hrthm.2010.07.027

10. Patocskai B, Barajas-Martinez H, Hu D, Gurabi Z, Koncz I, Antzelevitch C. Cellular and ionic mechanisms underlying the effects of cilostazol, milrinone, and isoproterenol to suppress arrhythmogenesis in an experimental model of early repolarization syndrome. Heart Rhythm. 2016;13(6):1326-34. https://doi.org/10.1016/j.hrthm.2016.01.024 